
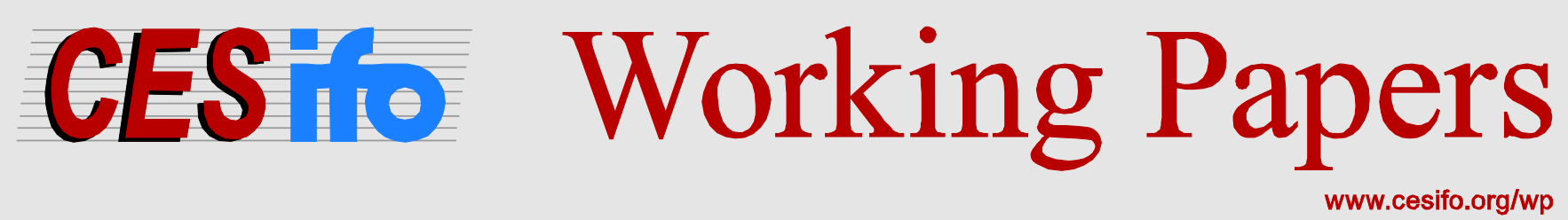

\title{
Intra-Group Heterogeneity in Collective Contests
}

\author{
Shmuel Nitzan \\ Kaoru Ueda
}

CESIFO WORKING PAPER NO. 4096

CATEgory 2: Public CHOICE

JANUARY 2013
An electronic version of the paper may be downloaded
- from the SSRN website: Www.SSRN.com
- from the RePEc website: Www.RePEc.org
- from the CESifo website: www.CESifo-group.org/wp

\section{CESifo}




\title{
Intra-Group Heterogeneity in Collective Contests
}

\begin{abstract}
Collective contests are examined permitting heterogeneity of stakes within every competing group. Our first concern is whether unequal distribution of stakes in a group can enhance its win probability. Our second concern is whether a large stake in a group can be individually disadvantageous. We find that if a contest is sufficiently "hard" for a group, the answers to these questions are positive under plausible conditions. In such situations, monopoly of the private stake is most effective in enhancing the group's performance. Ironically, in the same cases, the welfare of many group members would be negatively related to the size of their individual stake.
\end{abstract}

JEL-Code: D700, D710, D720.

Keywords: collective contest, intra-group heterogeneity, elasticity of marginal effort costs, unequal stakes and group performance, military alliance, 'exploitation of the large'.

Shmuel Nitzan
Department of Economics
Bar Ilan University
Ramat Gan
52900 Israel
nitzans@mail.biu.ac.il

Shmuel Nitzan

partment of Economics

Ramat Gan

nitzans@mail.biu.ac.il 


\section{Introduction}

In many examples of contests, lobbying, litigation, patent races, ethnic conflicts, and so on, the unit competing for the prize is actually a group; members of a competing group win or lose the prize together. But the individuals usually have different positions in a group; politically, economically and culturally. Such intra-group heterogeneity can be reflected in the different valuations of the prize by the members.

In some cases, the prize is a private good which once won by one of the competing groups is contested again within that group. The result of this second-stage within-group contest is affected by the power structure of the group. The people politically dominant in the group usually have an advantage in competing on their share in the group stake. Expecting larger gain than other group members, they therefore tend to evaluate higher the winning of the prize contested in the first-stage of the betweengroup contest. ${ }^{4} \mathrm{~A}$ change in the political relationships among its members results in different distributions of interests with respect to the collective contest. Note that even when the prize is a group-specific public good, members in a group may evaluate it differently. Consider, for example, several local governments competing for a budget earmarked to subsidize elementary schools. Then a family with more children has more interest in the contest. On a choice of location for public facilities with positive or negative externalities to the selected locality (a stadium for the Olympic games, a waste treatment center, etc.), the residents of the selected area differ in their interest, dependent on their distance to the public facility. A conflict between different ethnicities or different religious groups is another interesting example, as Esteban and Ray (2011) argue; such a conflict often implies a contest between their principles, though the financial value of the competed prize itself also matters. In this contest, a member more committed to the value judgments of the group (i.e., a more radical member) has higher appreciation for the prize. The intra-group heterogeneity of stakes from the prize is due to such reasons. Our purpose is to examine the implications of such heterogeneity ${ }^{5}$.

\footnotetext{
${ }^{4}$ We can apply a modified version of the two-stage model studied in Katz and Tokatlidu (1998) to describe the case. The effort cost function of an individual in each stage is assumed to be nonlinear (increasing marginal costs), and the contest success functions assigned to each member for the second-stage withingroup contest are asymmetric, reflecting the different political power of the members.

${ }^{5}$ The typical assumption in the literature on collective contests has been that the individuals are symmetric within each of the competing groups. The effect of heterogeneity has been mainly confined to differences
} 
One can ask whether such dissimilarity among the members is good or bad for a competing group. The answer to this question is equivocal. In their model of conflict concerning an ethnic policies, Esteban and Ray (2011b) show that more equal distribution of radicalism induces an ethnic group to be more active in the conflict and, in turn, to attain a higher win probability. Epstein and Mealem (2009) study a collective contest where an interest group enhances its win probability by expansions of membership that equalize the intra-group distribution of stakes, rather than by expansions that make it more unequal. These recent results establishing that a group with similar members is more active in a collective contest are contrary to the classical conjectures of Olson(1965) on the "logic of collective action," according to which a group whose members have highly unequal interests in the collective action will be more active. This conjecture is valid, however, in the collective contest on group-specific public goods studied by Baik (2008). In equilibrium of his model, everyone except those with the highest valuation of the prize in each group chooses not to put effort. A direct consequence is that unequal distribution of stakes within a group induces large effort.

The contrasting answers to the question posed in the preceding paragraph are due to the difference in the assumed form of the marginal effort cost function. If each individual's marginal effort cost function is strictly convex, similar valuations of the prize by the group members enhance the win probability of the group. However, Baik's opposite results are directly obtained from the assumption that each individual has constant marginal costs. The existing arguments against or in-favor of intra-group heterogeneity should therefore be examined with caution because a considerable divergence exists between the assumed forms of the effort cost functions.

In this paper a different approach is taken. Assuming very weak and plausible restrictions on the form of the effort cost functions, we try to specify the situations in which unequal valuations of the prize by the group members enhance the win probability of the group. We will show that one such situation occurs when the conflict is "hard."

in group size or effort costs between groups, under alternative assumptions regarding the nature of the prize. Katz, et al. (1990) and Riaz, et al. (1995) have shown that a group with a larger membership does not attain a lower winning probability, if the contested prize is a group-specific pure public good. Considering a class of impure public-good prizes that includes the pure public-good prize and the pure private-good prize as special cases, Esteban and Ray (2001) derive a sufficient condition ensuring that larger groups have a higher winning probability. For a useful comprehensive survey of the literature, see Konrad (2009). 
Our precise definition of the term "a hard contest for a group" applies, in particular, to a contest with a large number of participating groups and to a contest where the group has a very strong opponent. If a group competes for a prize with sufficiently many rivals or with a very superior rival, unequal stakes among the members can enhance its performance.

Our approach also sheds new light on another aspect of intra-group heterogeneity, the so called "exploitation of the great by the small," Olson (1965). In a collective contest, an individual with a high stake does not necessarily attain a higher welfare than members of the same group who have low stakes. The reason is that a collective contest can be viewed as a number of intra-group collective-action problems embedded in a competitive environment; in every group, members have incentives to free-ride while attempting to win the prize. A large stake in a collective contest prompts an individual to be more active, and as a result, to be more vulnerable to free-riding by the other members in the same group. Since Olson and Zeckhauser (1966) argue that it explains an excessive burden of a large country in a military alliance, the problem of "exploitation of the great by the small" has been considered mainly as that of private provision to a public good in a group ${ }^{6}$. We have found, however, that the severity of conflict with the other groups (such conflict is an essential factor for military allies) determines the severity of the exploitation problem in a group. The harder a conflict for a group, the wider the range of the exploited members (i.e., those attaining lower utility than members with smaller stakes) in the group. To answer adequately the questions related to intra-group heterogeneity in collective contests, we need to take into account how hard the contest is for each group.

In the next section, we introduce an extension of the share function approach to aggregative games systematically developed by Cornes and Hartley $(2003,2005)^{7}$, to analyze our model of group contests with intra-group heterogeneity. This useful method clarifies the basic properties of equilibrium, and considerably facilitates the analysis in

\footnotetext{
${ }^{6}$ Sandler (1993) gives a detailed survey of this subject.

${ }^{7}$ In aggregative games, each player's payoff is determined by own strategy and the sum of the strategies of all players. A classic example of such games is Cournot oligopoly, and many models of contests by individuals also belong to this class of games. Esteban and Ray (2001) have already adopted this approach (even previous to Cornes and Hartley) to analyse group contests, but their treatment heavily depends on the assumption that the members in each competing group are homogeneous.
} 
the following sections. Section 3 examines our first problem, the relationship between dissimilar stakes among the members of a group and its performance in the contest. The precise meaning of "a hard contest" and some examples are provided. Section 4 derives a condition that determines the range of individuals in a group who are subjected to exploitation of the great by the small. Section 5 considers what happens if our restrictions on the form of the effort cost function are dismissed, in order to highlight the importance of these restrictions. The dismissal opens the route to an interesting proposition which can be called the "anti-Olson theorem." Concluding remarks are made in Section 6. All proofs are relegated to the last section.

\section{A collective contest with intra-group heterogeneity}

Consider a contest in which $m$ groups compete for a prize. The population of group $i$ is denoted by $N_{i}$. Individuals decide how much effort to put into the contest simultaneously and non-cooperatively. The individual contributions are aggregated in every group, and the group which wins the prize is determined on the basis of those aggregated group efforts.

To describe intra-group heterogeneity in the value of, or the stake from, the contested prize, we assume that it is a mixture of group-specific public goods and private goods. Actually, the model allows several specifications of the prize; a group specific public-good prize valued differently within a group, a divisible private-good prize competed through an asymmetric second-stage within-group contest, and so on. Here we adopt a moderately general specification that includes these cases. Denote the values of the public-good part and the private-good part of the prize for every competing group by $P \geq 0$ and $M>0$, respectively ${ }^{8}$. The sharing of the private-good part within a group could be unequal, reflecting the different positions of the members and/or the power structure in each group. For member $k$ of group $i$, the share of the private-good part of the prize is denoted by $y_{i k}\left(k=1, \cdots, N_{i}\right)$, where $\sum_{k=1}^{N_{i}} y_{i k}=M$. The public-good part and the privategood part of the prize are assumed to be perfect substitutes for the contestants. The stake for member $k$ of group $i$ is thus given by $v_{i k}=P+y_{i k}\left(k=1, \cdots, N_{i}\right)$. The stake vector of

\footnotetext{
${ }^{8}$ We can generalize the model to the case where $P$ and $M$ are different among competing groups, but such an extension does not change the main results.
} 
group $i, \mathbf{v}_{i}=\left(v_{i 1}, \ldots, v_{i N_{i}}\right)$, represents a distribution of the individual group members' interests in the contest ${ }^{9}$.

All members of a group are assumed to have the same form of the contest-effort cost function $c_{i}$, i.e., member $k$ of group $i$ bears the cost $c_{i}\left(a_{i k}\right)$ when putting the amount of effort $a_{i k} \geq 0$ into the contest ${ }^{10}$. The effort cost function $c_{i}$ satisfies $c_{i}(0)=0$, and is assumed to be twice continuously differentiable, $c_{i}^{\prime}(a)>0$ and $c_{i}^{\prime \prime}(a)>0$, for all $a>0$. We write $c_{i}^{\prime}(0)=\lim _{a \rightarrow 0} c_{i}^{\prime}(a)$. Let $\alpha_{i}(a)=\frac{c_{i}^{\prime \prime}(a)}{c_{i}^{\prime}(a)} a$ denote the elasticity of the marginal effort cost functions for members of group $i$. Notice that the cost function is assumed to be strictly convex, i.e., the marginal cost is strictly increasing, which is a necessary assumption for the uniqueness of equilibrium in the contest.

The winning probability of group $i$ is assumed to be determined by the lottery contest success function $\frac{A_{i}}{A}$, where $A_{i}=\sum_{k=1}^{N_{i}} a_{i k}$ is the effort of group $i$, and $A=\sum_{j=1}^{m} A_{j}$ is the total amount of effort made by all the competing groups. Each individual is assumed to be risk-neutral and the expected utility of member $k$ of group $i$ has the form: ${ }^{11}$

$$
E U_{i k}=\frac{A_{i}}{A} v_{i k}-c_{i}\left(a_{i k}\right) .
$$

Given the amounts of other players' efforts, this function is strictly concave with respect to $a_{i k}$. So the first-order necessary and sufficient condition for the best-response takes the form:

$$
\frac{A-A_{i}}{A^{2}} v_{i k}-c_{i}^{\prime}\left(a_{i k}\right) \leq 0,
$$

with equality holding for $a_{i k}>0$. Notice that the amounts of the other players' efforts appear only in aggregated forms in this condition. By exploiting this property, we can derive the device called the share functions, which are very useful in making the

\footnotetext{
${ }^{9}$ When we consider the model as a contest for a group specific public-good prize evaluated differently in a group, the stake $v_{i k}$ is directly interpreted as the valuation of the public-good by member $k$ of group $i$.

${ }^{10}$ Even if we allow the members in a same group to have different effort cost functions, the results in this section (the existence and uniqueness of equilibrium) are still true.

${ }^{11}$ When no group puts positive effort, we assume that each group attains the winning probability $1 / \mathrm{m}$. Then, every member $k$ in each group has the incentive to deviate by making a small positive effort because $c_{i}(0)=0<v_{i k}$. Thus $A=0$ cannot be the total effort in equilibrium. The same conclusion is derived even if we assume that the winning probability is 0 for each group when $A=0$.
} 
following analysis transparent;

Proposition 1. There exist functions $s_{i}\left(A, \mathbf{v}_{i}\right):(0, \infty) \times(0, \infty)^{N_{i}} \rightarrow[0,1) \quad(i=1, \cdots, m)$ satisfying the following properties; given a combination of stake vectors $\left(\mathbf{v}_{1}, \cdots, \mathbf{v}_{m}\right)$, $A^{*}>0$ is the total amount of effort in a (pure-strategy) Nash equilibrium if and only if $\sum_{i=1}^{m} s_{i}\left(A^{*}, \mathbf{v}_{i}\right)=1$. For each group $i$, the corresponding equilibrium level of effort denoted by $A_{i}^{*} \geq 0(i=1, \cdots m)$ is derived from the equation $\frac{A_{i}^{*}}{A^{*}}=s_{i}\left(A^{*}, \mathbf{v}_{i}\right)$. Furthermore,

(a) $s_{i}$ is continuous with respect to $A>0$,

(b) $s_{i}$ is strictly decreasing in A as long as it takes a positive value, and

(c) $\lim _{A \rightarrow 0} s_{i}\left(A, \mathbf{v}_{i}\right)=1$ and $\lim _{A \rightarrow \infty} s_{i}\left(A, \mathbf{v}_{i}\right)=0$,

for all $i=1, \cdots, m$.

We call the function $s_{i}$ the share function of group $i$.

By properties (a) and (b) of the share functions, we can see that $\sum_{i=1}^{m} s_{i}\left(A, \mathbf{v}_{i}\right)$ is continuous and strictly decreasing in $A$ as long as it takes a positive value. Property (c) implies that both $\lim _{A \rightarrow 0} \sum_{i=1}^{m} S_{i}\left(A, \mathbf{v}_{i}\right)=m$ and $\lim _{A \rightarrow \infty} \sum_{i=1}^{m} S_{i}\left(A, \mathbf{v}_{i}\right)=0$. It should be clear from the Intermediate Value Theorem that these observations establish the existence and uniqueness of a pure-strategy Nash equilibrium ${ }^{12}$. Since $0 \leq s_{i}\left(A, \mathbf{v}_{i}\right)<1$ for all $i$, at least two groups make a positive effort in equilibrium. These results are summarized as follows:

Corollary. Given a combination of stake vectors $\left(\mathbf{v}_{1}, \cdots, \mathbf{v}_{m}\right)$, there exists a unique (purestrategy ) Nash equilibrium of the contest. In equilibrium, at least two groups choose positive levels of effort.

\footnotetext{
${ }^{12}$ Ryvkin (2011) uses another approach to derive existence and uniqueness of equilibrium for a contest model similar to ours. But his proof seems to critically depend on the restriction that the limit of the marginal effort cost at the zero effort level is not positive, i.e. $c_{i}^{\prime}(0)=0$ in terms of our notation. On the other hand, our main arguments are related to the case of $c_{i}^{\prime}(0)>0$. In fact we need the existence and
} 
Since the marginal cost function $c_{i}^{\prime}(a)$ is assumed to be strictly increasing, the continuous inverse function $\left(c_{i}^{\prime}\right)^{-1}$ is well defined. We can therefore specify the equilibrium contribution of member $k$ in group $i, a_{i k}{ }^{*}$, by the first-order condition (2), as follows:

$$
a_{i k}^{*}=\varphi_{i}\left(\frac{1-s_{i}\left(A^{*}, \mathbf{v}_{i}\right)}{A^{*}} v_{i k}\right),
$$

where

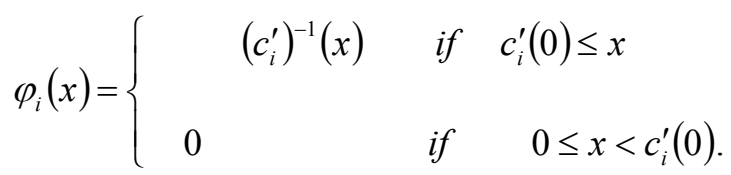

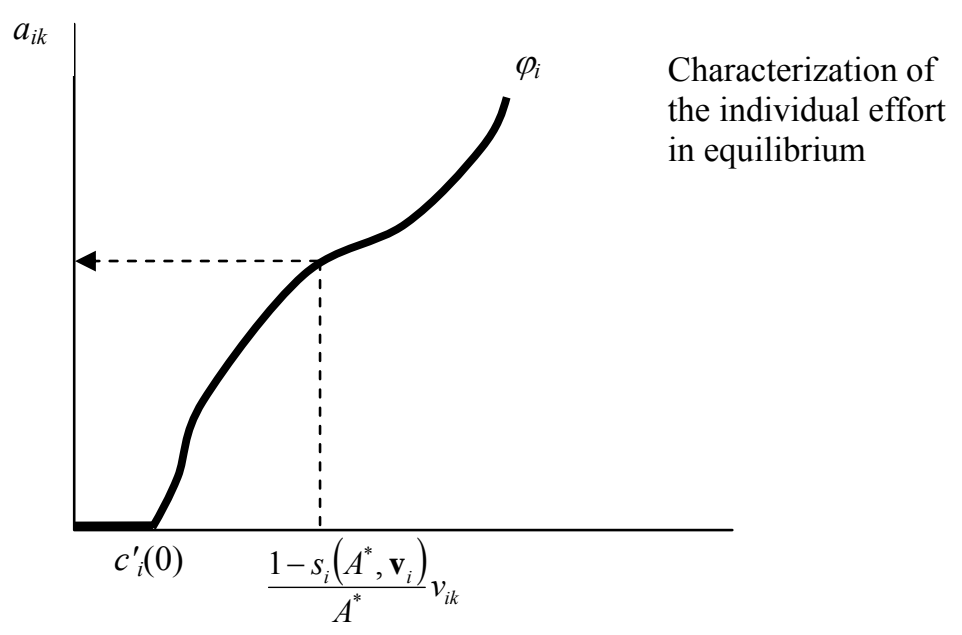

Figure 1

This characterization is presented in Figure 1, which shows that the individual contribution in a group is increasing with the stake from the contest. We can also deduce from this figure that, as long as $c_{i}^{\prime}(0)=0$ holds, a member of group $i$ always makes a positive effort. Non-contributors, i.e., individuals putting no effort, can exist only in the case where $c_{i}^{\prime}(0)>0$ holds. To allow for the usual occurrence of obstinate mobilization problems in real collective actions, we will treat the latter case as our main case. Let us 
refer to the inequality as the "potential non-contributor" condition, or

Condition PNC: $c_{i}^{\prime}(0)>0$ for all $i$.

To derive the results in the next two sections, we further introduce the following regularity condition on the effort cost functions;

Condition RC: $\lim _{a \rightarrow 0} c_{i}^{\prime \prime}(a) a=0$ for all $i$.

It is an innocuous condition covering a very wide range of effort cost functions. Unless the second derivative of $c_{i}$ goes to infinity as the effort level approaches zero, this condition trivially holds. Furthermore, an important special class of cost functions, those with constant elasticity of marginal cost, also satisfies the condition. ${ }^{13}$

\section{Unequal stakes and group performance}

Let us examine whether intra-group heterogeneity of prize-valuations in a group is good or bad for raising its win probability in a collective contest. We accomplish this task by comparison of different stake vectors set on a group. Consider two possible such vectors; in one vector certain members get a very large gain and the other members get a very small one, while in the other vector all members have moderate more even gains. The question here is: does the first distribution make the group more effective in the contest than the second less biased distribution?

To consider this question, we will restrict our arguments to cases where the distributions represented by the stake vectors can be ordered according to the relation of Lorenz domination. Take two stake vectors $\mathbf{v}_{i}=\left(v_{i 1}, \ldots ., v_{i N_{i}}\right)$ and $\mathbf{v}_{i}^{\prime}=\left(v_{i 1}^{\prime}, \ldots, v_{i N_{i}}^{\prime}\right)$, and assume that, without loss of generality, $v_{i 1} \leq \ldots \leq v_{i N_{i}}$ and $v_{i 1}^{\prime} \leq \ldots \leq v_{i N_{i}}^{\prime}$. The latter vector (or the represented distribution of valuations) is called more unequal than the former in the sense of Lorenz dominance (or Lorenz worsening) if $\sum_{k=1}^{h} v_{i k} \geq \sum_{k=1}^{h} v_{i k}^{\prime}$, for all $h \leq N_{i}$,

\footnotetext{
${ }^{13}$ This is the case where $c_{i}(a)=K a^{\alpha+1}$ with $K$ and $\alpha$ being positive constants. Then the marginal effort cost function is strictly convex if and only if $\alpha>1$.
} 
with strict inequality for at least one $h$, and $\sum_{k=1}^{N_{i}} v_{i k}=\sum_{k=1}^{N_{i}} v_{i k}^{\prime}{ }^{14}$. Dasgupta et al. (1973) prove that the following three statements are equivalent: (i) $\mathbf{v}_{i}{ }^{\prime}$ is more unequal than $\mathbf{v}_{i}$ in the sense of Lorenz dominance. (ii) $\mathbf{v}_{i}$ can be obtained from $\mathbf{v}_{i}{ }^{\prime}$ by a finite sequence of transformations of the form $v_{i l}(t+1)=v_{i l}(t)+\varepsilon(t) \leq v_{i h}(t), v_{i h}(t+1)=v_{i h}(t)-\varepsilon(t) \geq v_{i l}(t)$ with $\varepsilon(t)>0$ for some $h$ and $l(h>l)$, and $v_{i k}(t+1)=v_{i k}(t)$ for all $k \neq h, l$. (iii) For any strictly convex function $f$ defined on the set of real numbers, we have $\sum_{k=1}^{N_{i}} f\left(v_{i k}\right)<\sum_{k=1}^{N_{i}} f\left(v_{i k}^{\prime}\right)$.

Our first main result can now be stated.

Proposition 2. Consider a competing group $j$ whose marginal effort cost function satisfies the conditions $P N C$ and $R C$. There exists a positive value $A^{M}(j)$ for the group with the following properties: assume that the total effort $A^{*}$ is larger than or equal to $A^{M}(j)$ and at least one member of the group makes a positive contribution in equilibrium. Then,

(a) If at least two members in group $i$ have positive shares in the private-part of the prize, the winning probability can be raised by a Lorenz worsening change in the stake vector. This change also further enhances the equilibrium total effort, and

(b) The Lorenz worst stake vector, i.e. the monopoly in the private-part of the prize, maximizes the win probability of the group.

We will say that a contest is "hard" for group $j$ if the equilibrium total amount of effort is larger than or equal to $A^{M}(j)$. This term refers to the endogenous variable influenced by the choice of the group in question itself, and seems puzzling at first glance. But we can use the share functions to visualize how the outside environment of a group affects the total amount of effort, and such an observation helps us to find the structural factors making a contest hard for the group. Consider Figure 2 which presents the schedules of the share function of group $j$ and that of the sum of the share functions. Since the equilibrium total amount of effort is determined by the equation $\sum_{i=1}^{m} s_{i}\left(A^{*}, \mathbf{v}_{i}\right)=1$, a rise of the total equilibrium effort is obtained if and only if the

\footnotetext{
${ }^{14}$ Since $\sum_{k=1}^{N_{i}} v_{i k}=\sum_{k=1}^{N_{i}} v_{i k}^{\prime}=P+M$, the last condition is redundantly held in our model.
} 
schedule of $\sum_{i=1}^{m} s_{i}\left(A, \mathbf{v}_{i}\right)$ shifts up, as the dotted curve in the figure. Any structural factor of a contest external to the group ${ }^{15}$ inducing such a shift, therefore, makes the contest harder for the group. We can immediately give two examples of such factors; the number of competing groups and the superiority of the opponents. As more groups take part in the contest, the schedule of the summed share functions moves upward. Also, if the share function of a powered opponent shifts up, so does the sum. To confirm that our argument on a "hard contest" is sound, let us examine the effect of these changes formally.

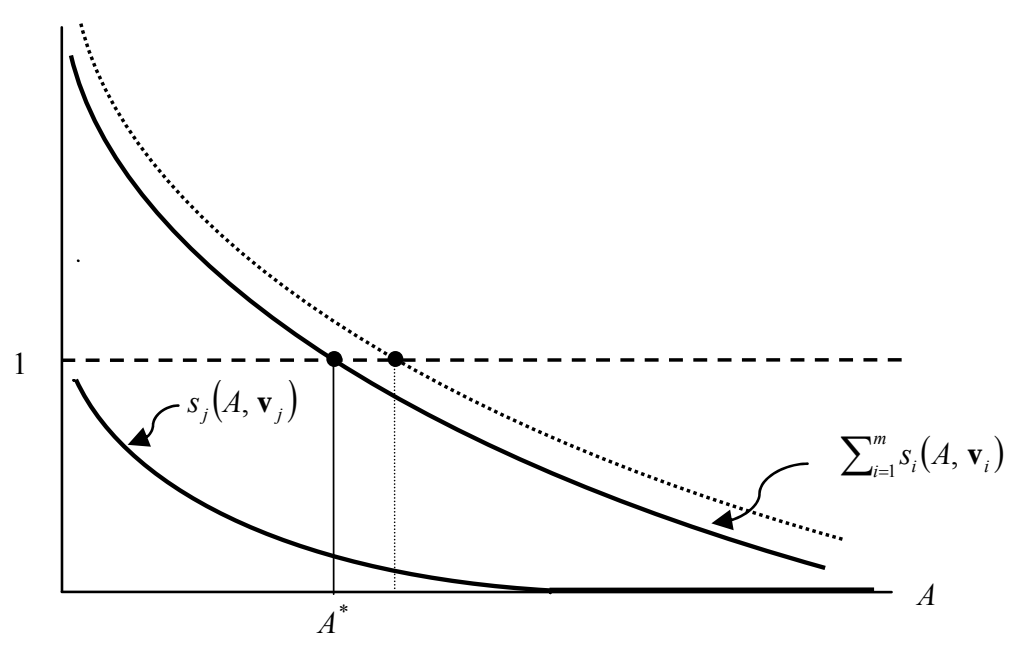

Figure 2

Fix the conditions of group 1, i.e. the group size $N_{1}$, the stake vector $\mathbf{v}_{1}$ and the effort cost function $c_{1}(a)$ for each member. Assume further that its share function $s_{1}$ satisfies the inequality $s_{1}\left(A^{M}(1), \mathbf{v}_{1}\right)>0{ }^{16}$. Then, we have the following two stories.

Many competing groups: Suppose that all competing groups in the contest are replicas of group 1 and that the number of the groups is $m$. The share function of each group is the

\footnotetext{
${ }^{15}$ Notice that the share function of the group itself does not move as long as its properties (effort cost functions of the members and the stake vector) are constant.

${ }^{16}$ Such a case is real. For example, let $N_{1}=3, P=11, M=24$, and the private-good prize be equally divided in group 1. If the effort cost function has the form $c_{1}(a)=\frac{a^{3}}{3}+\frac{3}{2} a$, we can set $A^{M}(1)=12$ (see the
} 
same as $S_{1}\left(A, \mathbf{v}_{1}\right)$, and the equilibrium total amount of effort $A^{*}$ is determined by the equation $s_{1}\left(A^{*}, \mathbf{v}_{1}\right)=\frac{1}{m}$. Since the share function is continuous, $A^{*}$ exceeds $A^{M}$ when $m$ is large enough. This argument can be generalized to the case where the competing groups are different.

Superior opponents: Suppose that the prize is competed by groups 1 and 2, and the latter group has the same size and stake vector as the former, i.e. $N_{1}=N_{2}=N$ and $\mathbf{v}_{1}=\mathbf{v}_{2}=\mathbf{v}$. But the effort cost function for individuals in group 2 has the form $\theta \cdot c_{1}(a)$, where $0<\theta<1$ is a parameter representing the superiority of group 2 (the cost advantage of individuals in group 2 relative to members of group 1 in making efforts) ${ }^{17}$. Now, consider what happens if the equilibrium total effort $A^{*}$ does not exceeds $A^{M}(1)$ for any $\theta$. Since $s_{1}\left(A^{M}(1), \mathbf{v}_{1}\right)>0, A^{*} \leq A^{M}(1)$ implies that at least one individual of group 1 chooses a positive amount of effort in equilibrium. Let this person's valuation of the prize be $w$. By construction, there exists an individual $k$ in group 2 who has the same valuation. We can therefore use equations (3) and (4) to establish the following relations between the equilibrium amounts of effort by this person.

$$
a_{2 k}^{*}=\left(c_{1}^{\prime}\right)^{-1}\left(\frac{s_{1}\left(A^{*}, \mathbf{v}_{1}\right)}{A^{*} \cdot \theta} w\right) \geq\left(c_{1}^{\prime}\right)^{-1}\left(\frac{s_{1}\left(A^{M}(1), \mathbf{v}_{1}\right)}{A^{M}(1) \cdot \theta} w\right) .
$$

It is possible to set $\theta<\frac{s_{1}\left(A^{M}(1), \mathbf{v}_{1}\right) \cdot w}{A^{M}(1) \cdot c_{1}^{\prime}\left(A^{M}(1)\right)}$, which yields a contradiction, namely, that the individual's chosen effort is larger than the total amount. Hence the equilibrium amount of effort must exceed $A^{M}(1)$ if $\theta$ is small enough. Again, we can generalize this argument to the case where group 2 is different from group 1 in its size and stake vector.

We can also translate the above cost superiority condition into superiority stated in terms of income or political influence. To introduce income variability among groups, we modify the model by assuming that an individual's preferences are represented by an

proof of Proposition 2 for the detail), while the share function has positive values until $A$ reaches to $\frac{38}{3}$.

${ }^{17}$ In the same framework, we can treat the case where excessive power of rivals is due to higher rival stakes. Let the effort cost function have the same form in group 2 as group 1 , but $\mathbf{v}_{2}=\frac{\mathbf{v}_{1}}{\theta}$. Then the game 
additively separable utility function of his benefit from the prize and of his income $I$. Specifically, the individual's utility is $u+V(I)$, where $u$ and $V$ are the benefits from the prize and income, respectively. Let $V^{\prime}(I)>0$ and $V^{\prime \prime}(I)<0$ for all $I>0$. Interpreting the effort level $a$ as money expenditure, we can define the cost function of a member in group $i$ as $v_{i}(a)=-V\left(I_{i}-a\right)$, where $I_{i}$ is the common income of the members of group $i$. Then the groups containing richer individuals have lower marginal effort costs. To introduce difference among groups in terms of political influence or lobbying power of the individuals, $A_{i}$ has to be interpreted as aggregate effort measured by an efficiency unit, and assume that each individual of group $i$ needs to make $e_{i} a\left(e_{i}>0\right)$ units of effort to produce the efficiency units $a$. The cost function will therefore have the form $v_{i}\left(e_{i} a\right)$ and differences in political capabilities among groups can be transformed to differences in the marginal effort costs.

The meaning of Proposition 2 is understandable if one imagines a situation where the winning probability of a group is very low in a hard contest. Each member has bad prospects regarding the winning of his group, and consequently makes a very low contribution. Even if a member becomes a non-contributor due to a shift of interest from him to another member, the decline in the group effort is very small, and further shift only increases the group effort. It should be emphasized, on the other hand, that the proposition does not require the actual existence of non-contributors. If a group contains a non-contributor at the outset, a shift of stake from non-contributors to other members has a decisive positive effect on the win probability of their group ${ }^{18}$. But the validity of Proposition 2 is not restricted to such a special case, and can be applied even to the case that all individuals share the same stake and therefore put the same amount of effort. This is the reason why we can conclude that monopoly of the private-part prize is best for enhancing the winning probability of the group.

\footnotetext{
is equivalent to that in the text, but now $\theta$ is the parameter of the stake-size in group 2 .

18 This is analogous to the arguments made by Bergstrom et al. (1986) in the context of voluntary provision of public goods. Under the assumption that the marginal rate of transformation between the private and public goods is unity, they have shown that an income transfer among individuals affects the provision of public goods if it is made between existing contributors and non-contributors. In particular, they have proved that equalizing income redistributions that involve any transfers from contributors to noncontributors will decrease the equilibrium supply of the public good.
} 


\section{Unequal stakes and "exploitation of the great by the small"}

Intra-group heterogeneity of stakes from the prize matters not only for the performance of a group, but also for the welfare of its members. Here the problem is that a larger stake of an individual is not necessarily associated with a higher expected utility in equilibrium. A large stake in a collective contest works two-ways for the well-being of an individual in a group. While the promised gain from winning is large, it makes an individual more eager to be active, and as a result, more vulnerable to the other members' free-riding. Olson (1965) argues that the latter effect is so serious that a large stake from a collective action in a group is eventually disadvantageous for the member ${ }^{19}$, and calls this conjecture the "exploitation of the great by the small". By equation (3), we can confirm that a large stake in fact induces much equilibrium effort by an individual. And the condition that appears in the next proposition enables us to examine whether the "exploitation of the great by the small" actually happens in equilibrium.

Proposition 3. Consider members $l$ and $h$ of group $j$ with $v_{j l}<v_{j h}$. Let $s_{j}{ }^{*}$ be the equilibrium value of the share function and $A_{j}{ }^{*}$ the equilibrium effort of group $j$. If

$$
\alpha_{j}\left(\varphi_{j}\left(\frac{1-s_{j}^{*}}{A^{*}} v\right)\right) \underset{(>)}{<} \frac{1-s_{j}^{*}}{A_{j}^{*}} \varphi_{j}\left(\frac{1-s_{j}^{*}}{A^{*}} v\right)
$$

holds for all $v$ in the interval $\left(v_{j l}, v_{j h}\right)$, then the expected utility of member $h$ is smaller (larger) than that of member $l$.

When inequality (5) holds, therefore, having a larger stake in the contest than

\footnotetext{
${ }^{19}$ In the collective contests with constant marginal costs discussed by Baik (2008), it is not unusual that the expected utilities of the highest-valuation members are the lowest. Due to the assumption of constant marginal costs, only the highest-valuation members in each group are the contributors. We can therefore easily construct such examples. Let member $h$ have the highest valuation of the prize in group $j$, and let $v_{j l}<v_{j h}$ for member $l$. Denote the constant marginal cost for the members in group $j$ by $\chi_{j}$. Also, let $a_{j h}^{*}>0$ be the equilibrium effort level of member $h$, and $p_{j}{ }^{*}$ the winning probability of group $j$. Member $l$ ends as a non-contributor. If the expected utility of member $h, p_{j}^{*} v_{j h}-\chi_{j} a_{j h}^{*}$, is higher than that of member $l$, $p_{j}{ }^{*} v_{j l}$, raise the value of $v_{j l}$ keeping the inequality $v_{j l}<v_{j h}$. Since this operation does not change any individual's effort level, we can eventually reach a situation in which member $l$ attains a higher expected utility than member $h$.
} 
another member is undesirable, and everyone wants to change off ${ }^{20}$. In this sense, the conjecture on the "exploitation" is supported. We can see that $\alpha_{j}$, the size of the elasticity of the marginal effort costs, plays an important role in determining whether reversals of stakes and expected utilities among individuals in a group happen or not. The reason is clear if we notice that $\alpha_{j}$ is the reciprocal of the elasticity of $\varphi_{j}$, which is the function relating the stake of an individual and the equilibrium contribution. If $\alpha_{j}$ is very small (large), the elasticity of $\varphi_{j}$ is very large (small) and the increment of effort induced by a rise in the stake is sufficient (insufficient) to cause the reversal. But it is possible to derive more implications from Proposition 3. Consider for each member his/her share of effort in the group. It turns out that the average is the reciprocal of its membership, and the standard score for member $k$ of group $j$ is given by

$$
\frac{\left(a_{j k} / A_{j}\right)-\left(1 / N_{j}\right)}{S D_{j}}
$$

where $S D_{j}$ is the standard deviation of the effort shares in group $j$. Suppose that there exists a real number $\xi$ such that

$$
\sup _{k} \alpha_{j}\left(\varphi_{j}\left(\frac{1-s_{j}^{*}}{A^{*}} v_{j k}\right)\right) \leq\left(S D_{j} \cdot \xi+\frac{1}{N_{j}}\right)\left(1-s_{j}^{*}\right) .
$$

Proposition 3 then implies that the expected utility decreases with the size of the stake from the prize among the individuals whose standard score of the effort share is higher than or equal to $\xi$. Inequality (6) reveals that the "exploitation of the great by the small" occurs most often among members with large stakes from the prize, whose effort levels are relatively high. Furthermore, we can see from the inequality what factors influence the range of individuals exposed to the "exploitation." The range expands when the elasticity of the marginal cost of effort is low, when the equilibrium winning probability of the group is low, when the group-size is small, and when the standard deviation of the effort shares is large ${ }^{21}$.

We find, again, that the total amount of effort in equilibrium is a significant variable, which affects two of the factors in (6). When it is high, $s_{j}^{*}$ is low by Proposition

\footnotetext{
${ }^{20}$ Notice that this argument does not require interpersonal comparison of utilities.

${ }^{21}$ As in the arguments in the last section, we do not need the actual existence of non-contributors to derive the results here. This is in sharp contrast to the derivation of the results in Baik (2008) and Olson and
} 
1, and $\sup _{k} \alpha_{j}\left(\varphi_{j}\left(\frac{1-s_{j}^{*}}{A^{*}} v_{j k}\right)\right)$ is also low. Both effects make the conjecture on the "exploitation of the great by the small" more plausible. As a conflict gets harder for a group, the range of individuals in a group who are exposed to the "exploitation" is expanded where those with a larger stake have a lower expected utility. This general observation enables us to derive the following corollary of Proposition 3.

Corollary. Pick a competing group $j$ and let the marginal effort cost function $c_{j}^{\prime}$ satisfy the conditions PNC and RC. There exists a positive value $A^{E}$ for the group such that, if the equilibrium total effort $A^{*}$ is larger than or equal to $A^{E}$, then the expected utility declines with the size of the stake from the prize among the individuals in group $j$ whose effort level is larger than or equal to the average of the group.

We have found another version of a "hard" contest for a group, in which everyone contributing more than the average of the group is exploited. Using the same reasoning as in the last section, we could argue that the exploitation of the great by the small is expected for a wide range of group members, if the contest is sufficiently competitive or the group is very inferior to its opponents. Furthermore, if a group includes actual noncontributors in equilibrium, which is rather incidental to a hard contest, the sub-group of those contributing more than the average could cover all members who make a positive contribution. In such a case, all the positive contributors are exposed to exploitation.

\section{The "anti-Olson Theorem"}

Our main results on intra-group heterogeneity are derived under the assumption of potential non-contributors, $c_{i}^{\prime}(0)>0$ for all $i$. Although it seems a trivial local condition making little difference, the condition marks where the implications of intra-group heterogeneity start to change. Notice at first that negation of the condition PNC, or assuming that $c_{i}^{\prime}(0)=0$, opens the way to the next proposition.

Zeckhauser (1966). 


\section{Proposition 4.}

For a group $j$, let $c_{j}^{\prime}(0)=0$ hold and the marginal cost function $c_{j}{ }^{\prime}$ be strictly convex (concave). Then, a Lorenz worsening change in the stake vector of the individual group members decreases (increases) its win probability, and increases (decreases) those of the other groups as long as they put positive amounts of effort in the initial equilibrium. Furthermore, all individuals in such other groups are better (worse) off.

Existing arguments on intra-group heterogeneity and performance are essentially based on this result, paying attention to the case of convex marginal costs. The condition of convex marginal effort costs or its equivalences are so convenient to derive clear results, that they are often used in the recent literature ${ }^{22}$. Once it is combined with the negation of the condition PNC, however, a Lorenz inferior distribution of stakes always weakens a group in a contest. And this combination has further serious implications. Since the right-hand-side of inequality (5) is always less than one, Proposition 3 implies that if the elasticity of the marginal effort cost functions in a group is always not less than one, the expected utility of the members is strictly increasing with the stake. When the marginal cost function in group $j$ is strictly convex and $c_{j}^{\prime}(0)=0$, however, $\inf _{a>0} \alpha_{j}(a) \geq 1$ always holds. Then, the "exploitation of the great by the small" never happens in the group. Furthermore, as Esteban and Ray show (2001, Proposition 2), this inequality is also sufficient to ensure that a group always attains a higher win probability by expanding its size, provided that the private prize won is allocated equally (notice that, by Proposition 4, it gives the stake vector attaining the highest win probability). Summarizing the results, we obtain the following statement, which could be called the anti-Olson theorem because its assertions invalidate the major conjectures on collective actions made by Olson ${ }^{23}$.

\footnotetext{
${ }^{22}$ We could refer to two articles mentioned in the introduction. Also, Ryvkin (2011) uses the property in examining how to sort individuals in competing groups to maximize the total effort. See Esteban and Ray $(1999,2011 \mathrm{a})$ on other important applications of this assumption.

${ }^{23}$ For a comprehensive list of these conjectures, see Sandler (1992).
} 


\section{The anti-Olson theorem.}

Let $c_{i}^{\prime}(0)=0$ and the marginal cost function $c_{i}{ }^{\prime}$ be strictly convex for every $i=1, \cdots, m$, then:

(i). A group attains a higher win probability with more equal distribution (in the sense of Lorenz domination) of the gains from the prize.

(ii). The larger an individual's stake in winning the contest, the higher his/her expected utility within a group.

(iii). The larger a group expands, the higher its probability of winning the contest (provided that the distribution of the stakes in each group is set to attain the highest win probability).

This theorem is clear and comprehensive, and tells us how we can construct a contest model without being concerned by the paradoxical outcomes suggested by Olson. But our results in the previous sections show that the validity of the above theorem is immediately eroded by just introducing the condition PNC. Unequal distribution of stakes could be advantageous, or the exploitation of the great could happen, in a group competing for a prize. Also the group size could be disadvantageous ${ }^{24}$. Unless we have a special reason to exclude the possibility at the outset that some individuals choose to be non-contributors, we should give up comprehensive clear cut conclusions on the effect of intra-group heterogeneity and carefully take into account the particular situation of the group.

\section{Concluding remarks}

When groups compete for a prize, the positions of their members are usually not even. As members of the same group they seek the prize collectively, but their stakes from winning are not necessarily equal. We have constructed a model of collective contests permitting intra-group heterogeneity of stakes from an impure public-good prize, and examined how such heterogeneity influences the win probability of a group and the welfare of its members. We have found that, if a contest is hard enough for a group, an

\footnotetext{
${ }^{24}$ We can easily imagine the case where the private-part of the prize is thinly distributed among the very large number of group members, which makes the majority of them non-contributors.
} 
unequal distribution of stakes enhances its winning probability and a large stake turns to be disadvantageous for an individual. It is confirmed that such a hard contest occurs for the group if the number of competing group is very large or the opponent groups are very strong. When we consider the effect of intra-group heterogeneity within a group in collective contests, we need to carefully specify the situation of the group.

Our results about a hard contest contain an irony. When a contest is hard enough for a group, a change of the power structure that concentrates the stakes in a small elite sub-group can enhance the group's performance. However, those elite members offered large stakes will eventually sacrifice their welfare for the other free-riding members.

One may ask whether our results on intra-group heterogeneity can be reversed when a contest is "mild" for a group. At least with respect to the effects on win probabilities, the answer can be yes. Let the marginal effort cost function of a group be convex. And also assume that the total amount of effort in equilibrium is so small, that even an individual group member who does not receive any share in the private-part of the prize makes positive contributions. In such a case, we can in effect apply Proposition 4. Then we could have a model that has two different phases for a group, depending on the size of the equilibrium total amount of effort; if it is large enough, monopoly of the private-part of the prize is the best distribution of the stake to win. If it is small enough, uniform distribution is the best.

An interesting open problem is the effect of group sharing rules under intragroup heterogeneity. Prior to the contest, members of a group may commit to a group sharing rule specifying how (at least part of) the won prize is distributed among them. If such a rule distributes more of the prize to those contributing more, it works as an incentive scheme for the members ${ }^{25}$. A group sharing rule operates impersonally, i.e., it does not assign any significance to the names of the group members. It specifies a transfer to an individual that hinges only on his/her behavior. If there is no intra-group heterogeneity, therefore, everyone in a group chooses the same amount of contribution in equilibrium as shown in the existing literature. If individuals are heterogeneous in a group, however, their reactions to a sharing rule must be different. Is a group sharing rule

\footnotetext{
${ }^{25}$ Nitzan (1991), Lee (1995), Ueda (2002), Baik and Lee (2007), and Nitzan and Ueda (2011) examine the working of group sharing rules as an incentive scheme in collective contests.
} 
more effective in raising the win probability for a group with more intra-group heterogeneity? Does it mitigate the exploitation of the great? These are questions to be answered in future research.

\section{Proofs}

\section{Proof of Proposition 1.}

Let us consider the following question regarding the effort of individual $k$ in group $i$, without complete specification of every other individual's effort level: if some $A$ and $A_{i}$ are the amounts, respectively, of total effort and of group $i$ 's effort in (a pure-strategy Nash) equilibrium, what value must $a_{i k}$ take? The answer to the above question is summarized as a continuous function $\rho_{i k}\left(A, A_{i}, v_{i k}\right)=\varphi_{i}\left(\frac{A-A_{i}}{A^{2}} v_{i k}\right)$ of $A>0, v_{i k}>0$ and $A_{i} \in[0, A]$, where $\varphi_{i}$ is the function defined by (4). We call this function the replacement function of member $k$ of group $i$, using the terminology of Cornes and Hartley (2003). In equilibrium, $a_{i k}=\rho_{i k}\left(A, A_{i}, v_{i k}\right)$ must hold for all $i=1, \ldots, m$ and $k=1, \ldots, N_{i}$, under the condition that $A_{i}=\sum_{i=1}^{N_{i}} a_{i k}$ for each $i$ and $A=\sum_{i=1}^{m} \sum_{i=1}^{N_{i}} a_{i k}$. Notice that the converse is also true in the model; the $a_{i k}$ 's satisfying all of these equations constitute an equilibrium configuration of effort levels in our collective contest.

We can see that $\rho_{i k}$ is a continuous function and, as long as it takes a positive value, strictly decreasing in $A_{i}$. Furthermore, as $A_{i}$ grows to $A$, its value shrinks to 0 (recall that $A$ and $A_{i}$ are independent variables in the individual replacement functions). Then the summation of $\rho_{i k} \mathrm{~s}$ ' over the members of group $i, \sum_{k=1}^{N_{i}} \rho_{i k}\left(A, A_{i}, v_{i k}\right)$, inherits those properties, and there exists a unique value $A_{i}^{*}$ such that $A_{i}^{*}=\sum_{k=1}^{N_{i}} \rho_{i k}\left(A, A_{i}^{*}, v_{i k}\right)$, where $0 \leq A_{i}^{*}<A$ always holds. This observation allows us to define a function $A_{i}\left(A, \mathbf{v}_{i}\right)$ satisfying the equation $A_{i}\left(A, \mathbf{v}_{i}\right)=\sum_{k=1}^{N_{i}} \rho_{i k}\left(A, A_{i}\left(A, \mathbf{v}_{i}\right), v_{i k}\right)$.

Now, we can define the share function of group $i$ by $s_{i}\left(A, \mathbf{v}_{i}\right)=\frac{A_{i}\left(A, \mathbf{v}_{i}\right)}{A}$. The conditions $A_{i}=s_{i}\left(A, \mathbf{v}_{i}\right) A$ for all $i$ and $\sum_{i=1}^{m} s_{i}\left(A, \mathbf{v}_{i}\right)=1$ are therefore equivalent to the equilibrium conditions $A_{i}=\sum_{k=1}^{N_{i}} \rho_{i k}\left(A, A_{i}, v_{i k}\right)$ and $A=\sum_{i=1}^{m} \sum_{k=1}^{N_{i}} \rho_{i k}\left(A, A_{i}, v_{i k}\right)$. 
Let us prove properties (a), (b) and (c) of the share function.

(a) If we can show that $A_{i}\left(A, \mathbf{v}_{i}\right)$ is continuous with respect to $A$ on $(0, \infty)$, this property is straightforward. Extend the domain of $\rho_{i k}$ with respect to $A_{i}$ by setting $\rho_{i k}\left(A, A_{i}, v_{i k}\right)=0$ for all $A_{i}>A$, at each $A>0$ and $0<v_{i k}$. We can see that $\gamma\left(A, A_{i}, \mathbf{v}_{i}\right)=\sum_{k=1}^{N_{i}} \rho_{i k}\left(A, A_{i}, v_{i k}\right)-A_{i}$ is a continuous function of $\left(A, A_{i}\right)$ on $(0, \infty) \times[0, \infty) . \Gamma=\left\{\left(A, A_{i}\right) \mid \gamma\left(A, A_{i}, \mathbf{v}_{i}\right)=0\right\}$ is, therefore, a closed set in this space. Suppose that $A_{i}\left(A, \mathbf{v}_{i}\right)$ is not continuous at $A^{0}>0$. We can find a positive number $\varepsilon_{0}$ and a sequence $\{A(n)\}$ in $(0, \infty)$ converging to $A^{0}$ with $\left|A_{i}\left(A(n), \mathbf{v}_{i}\right)-A_{i}\left(A^{0}, \mathbf{v}_{i}\right)\right| \geq \varepsilon_{0}$ for all $n$.

Since $0 \leq A_{i}\left(A(n), \mathbf{v}_{i}\right) \leq A(n)$ holds for all $n,\left\{A_{i}\left(A(n), \mathbf{v}_{i}\right)\right\}$ is a bounded sequence that has a convergent subsequence $\left\{A_{i}\left(A\left(n_{p}\right), \mathbf{v}_{i}\right)\right\}$. Denote its limit by $A_{i}{ }^{L}$. Then, $\left\{\left(A\left(n_{p}\right), A_{i}\left(A\left(n_{p}\right), \mathbf{v}_{i}\right)\right)\right\}$ is a converging sequence in a closed set $\Gamma$, and its limit belongs to $\Gamma$, i.e. $\gamma\left(A^{0}, A_{i}^{L}, \mathbf{v}_{i}\right)=0$. The value of $A_{i}$ satisfying this equation is unique, and $A_{i}^{L}=A_{i}\left(A^{0}, \mathbf{v}_{i}\right)$ must hold. This contradicts the construction of $\{A(n)\}$.

(b) Notice that $s_{i}\left(A, \mathbf{v}_{i}\right)$ is equal to $\sigma$ satisfying the equation

$$
\sigma=\frac{1}{A} \sum_{k=1}^{N_{i}} \varphi_{i}\left(\frac{1}{A}(1-\sigma) v_{i k}\right)
$$

and each $\varphi_{i}\left(\frac{1}{A}(1-\sigma) v_{i k}\right)$ is decreasing in $A$. In particular, it is strictly decreasing, as long as it takes a positive value. Hence the right-hand-side of the equation is strictly decreasing with $A$ as long as it takes a positive value and the desired result can be derived. (c) The range of $s_{i}\left(A, \mathbf{v}_{i}\right)$ is bounded, and $\sup _{A>0} s_{i}\left(A, \mathbf{v}_{i}\right) \leq 1$ exists. $\lim _{A \rightarrow 0} s_{i}\left(A, \mathbf{v}_{i}\right)$ is therefore equal to this value because $s_{i}\left(A, \mathbf{v}_{i}\right)$ is decreasing with $A$. We can write

$$
0=\lim _{A \rightarrow 0} A s_{i}\left(A, \mathbf{v}_{i}\right)=\lim _{A \rightarrow 0} \sum_{k=1}^{N_{i}} \varphi_{i}\left(\frac{1}{A}\left(1-s_{i}\left(A, \mathbf{v}_{i}\right)\right) v_{i k}\right) \geq \max _{k \in\left\{1, \ldots, N_{i}\right\}} \lim _{A \rightarrow 0} \varphi_{i}\left(\frac{1}{A}\left(1-s_{i}\left(A, \mathbf{v}_{i}\right)\right) v_{i k}\right) .
$$

If $\lim _{A \rightarrow 0} s_{i}\left(A, \mathbf{v}_{i}\right)<1, \lim _{A \rightarrow 0} \varphi_{i}\left(\frac{1}{A}\left(1-s_{i}\left(A, \mathbf{v}_{i}\right)\right) v_{i k}\right)=\lim _{x \rightarrow \infty} \varphi_{i}(x)$ holds for all $k$. As $\left(c_{i}^{\prime}\right)^{-1}$ is strictly increasing, $\lim _{x \rightarrow \infty} \varphi_{i}(x)$ must be positive and we get a contradiction to the above inequality. Hence, $\lim _{A \rightarrow 0} s_{i}\left(A, \mathbf{v}_{i}\right)=1$ must hold. For any value of $A$ larger than $1, \rho_{i k}\left(A, A_{i}, v_{i k}\right)$ does 
not exceed $\left(c_{i}^{\prime}\right)^{-1}\left(v_{i k}\right)$. Therefore, $A_{i}\left(A, \mathbf{v}_{i}\right)$ never exceeds $\sum_{k=1}^{N_{i}}\left(c_{i}^{\prime}\right)^{-1}\left(v_{i k}\right)$ and we obtain that $\lim _{A \rightarrow \infty} s_{i}\left(A, \mathbf{v}_{i}\right)=0$.

Q.E.D.

We use the following two lemmas to prove Proposition 2.

Lemma 1. Let $A^{*}$ be the total effort in equilibrium. Pick group $j$ and assume that its share function changes from $s_{j}(\cdot)$ to $s_{j}{ }^{v}(\cdot)$, possibly by a change in the stake vector, group size, and so on. Let this change result in total amount of effort $A^{* *}$ at the new equilibrium. If $s_{j}^{v}\left(A^{*}\right)>(<) s_{j}\left(A^{*}\right)$, or the value of the share function increases (decreases) at the initial equilibrium total effort, then $A^{* *}>(<) A^{*}$, and the winning probability of the group goes up (down). Furthermore, for any group $i$ other than $j$, the winning probability gets smaller (larger) as long as its aggregate effort is positive in the initial equilibrium. In addition, all members in a group such as $i$ are worse (better) off by the change.

\section{Proof of Lemma 1.}

Consider the case where $s_{j}{ }^{v}\left(A^{*}\right)>s_{j}\left(A^{*}\right)$. Suppose that $A^{*} \geq A^{* *}$. Then,

$$
\begin{aligned}
1=\sum_{i \neq j} s_{i}\left(A^{*}, \mathbf{v}_{i}\right)+s_{j}\left(A^{*}\right) & <\sum_{i \neq j} s_{i}\left(A^{*}, \mathbf{v}_{i}\right)+s_{j}{ }^{v}\left(A^{*}\right) \\
& \leq \sum_{i \neq j} s_{i}\left(A^{* *}, \mathbf{v}_{i}\right)+s_{j}{ }^{v}\left(A^{* *}\right)=1,
\end{aligned}
$$

and this contradiction requires that $A^{*}<A^{* *}$. We know that at least two groups put positive effort in equilibrium, and $s_{i}\left(A^{*}, \mathbf{v}_{i}\right)>0$ holds for some $i \neq j$. Since $s_{i}$ is strictly decreasing in a neighborhood of $A^{*}, s_{i}\left(A^{* *}, \mathbf{v}_{i}\right)<s_{i}\left(A^{*}, \mathbf{v}_{i}\right)$ holds. We, therefore, have for group $j$ that

$$
s_{j}\left(A^{*}\right)=1-\sum_{i \neq j} s_{i}\left(A^{*}, \mathbf{v}_{i}\right)<1-\sum_{i \neq j} s_{i}\left(A^{* *}, \mathbf{v}_{i}\right)=s_{j}{ }^{v}\left(A^{* *}\right) .
$$

Now, consider group $i$, other than $j$ which puts positive effort in the initial equilibrium (before the change in group $j$ ). If a member of the group is a non-contributor in both of the initial and the new equilibrium, his utility must decline because the winning probability of group $i$ declines. With the exception of such members, all members of the group change their effort levels in the same direction because 


$$
a_{i k}^{*}=\varphi_{i}\left(\frac{1-s_{i}\left(A^{*}, \mathbf{v}_{i}\right)}{A^{*}} v_{i k}\right) \text { for all } k=1, \cdots, N_{i} .
$$

If their contributions do not change or increase, they must become worse off. What remains to be examined is, therefore, the case where their contributions are lower in the new equilibrium. Denote the contribution by member $k$ in the initial and the new equilibrium, respectively, by $a_{i k}{ }^{*}$ and $a_{i k}{ }^{* *}$. Also, denote his/her expected utility in the initial and the new equilibrium, respectively, by $E U_{i k}{ }^{*}$ and $E U_{i k}{ }^{* *}$. For each member $h$ of group $i$ we therefore get that

$$
\left(A_{i}\left(A^{*}, \mathbf{v}_{i}\right)-a_{i h}^{*}\right)-\left(A_{i}\left(A^{* *}, \mathbf{v}_{i}\right)-a_{i h}^{* *}\right)=\sum_{k \neq h}\left(a_{i k}^{*}-a_{i k}^{* *}\right) \geq 0 .
$$

Since $a_{i h}^{* *}<a_{i h}^{*}$, we can see

$$
\begin{gathered}
E U_{i h}^{*}=\max \left\{\frac{A_{i}\left(A^{*}, \mathbf{v}_{i}\right)-a_{i h}^{*}+a_{i h}}{A^{*}-a_{i h}^{*}+a_{i h}} v_{i h}-c_{i h}\left(a_{i h}\right) \mid a_{i h} \geq 0\right\} \\
>\max \left\{\frac{A_{i}\left(A^{* *}, \mathbf{v}_{i}\right)-a_{i h}^{* *}+a_{i h}}{A^{* *}-a_{i h}^{* *}+a_{i h}} v_{i h}-c_{i h}\left(a_{i h}\right) \mid a_{i h} \geq 0\right\}=E U_{i h}^{* *} .
\end{gathered}
$$

The case of $s_{j}{ }^{v}\left(A^{*}\right)<s_{j}\left(A^{*}\right)$ can be proved similarly.

Q.E.D.

Lemma 2. Let $c_{j}$ satisfy the conditions $P N C$ and $R C$. Then, for any $0<\eta_{0}<c_{j}^{\prime}(0)$, there exists a level of effort $\hat{a}>0$ such that, given $0<\eta \leq \eta_{0}$, the function $\frac{c_{j}^{\prime}(a)-\eta}{a}$ is strictly decreasing on $(0, \hat{a}]$.

\section{Proof of Lemma 2.}

For any $0<\eta_{0}<c_{j}^{\prime}(0)$, the condition RC implies that there exists $\hat{a}>0$ such that for any $0<a \leq \hat{a}$

$$
c_{j}^{\prime \prime}(a) a<c_{j}^{\prime}(0)-\eta_{0} .
$$

Then, given $0<\eta \leq \eta_{0}$, it holds for any $0<a<\hat{a}$ that

$$
\frac{d}{d a}\left(\frac{c_{j}^{\prime}(a)-\eta}{a}\right)=\frac{1}{a^{2}}\left(c_{j}^{\prime \prime}(a) a-c_{j}^{\prime}(a)+\eta\right)<0
$$

\section{Proof of Proposition 2.}


Fix any $0<\eta_{0}<c_{j}^{\prime}(0)$. We have $\hat{a}>0$ described in Lemma 2. Denoting $x=c_{j}^{\prime}(a)-\eta$ for any $0<\eta \leq \eta_{0}$, we can see that $\frac{a}{c_{j}^{\prime}(a)-\eta}=\frac{\left(c_{j}^{\prime}\right)^{-1}(x+\eta)}{x}$ is strictly increasing with $x$ on the interval $\left(c_{j}^{\prime}(0)-\eta, \quad c_{j}^{\prime}(\hat{a})-\eta\right]$. Assume that the equilibrium total effort $A^{*}$ is large enough to satisfy $A^{*} \geq \max \left\{\frac{M}{c_{j}^{\prime}(\hat{a})-\eta_{0}}, \frac{P}{\eta_{0}}\right\}$.

Let $h$ and $l$ be members with $y_{j h,} y_{j l}>0$. Without loss of generality, we can assume that member $h$ makes a positive contribution in equilibrium, i.e. $\frac{1-s_{j}^{*}}{A^{*}} v_{j h}>c_{j}^{\prime}(0)$, where $s_{j}^{*}=s_{j}\left(A^{*}, \mathbf{v}_{j}\right)$. By definition of the stake, $\frac{1-s_{j}^{*}}{A^{*}} y_{j h}>c_{j}^{\prime}(0)-\frac{1-s_{j}^{*}}{A^{*}} P$ holds. On the other hand, $\frac{1-s_{j}^{*}}{A^{*}}\left(y_{j h}+y_{j l}\right)<c_{j}^{\prime}(\hat{a})-\eta_{0}<c_{j}^{\prime}(\hat{a})-\frac{1-s_{j}^{*}}{A^{*}} P$. Hence, by setting $\eta=\frac{1-s_{j}^{*}}{A^{*}} P$, we obtain the inequality:

$$
\frac{\left(c_{j}^{\prime}\right)^{-1}\left(\frac{1-s_{j}^{*}}{A^{*}}\left(y_{j h}+P\right)\right)}{\frac{1-s_{j}^{*}}{A^{*}} y_{j h}}<\frac{\left(c_{j}^{\prime}\right)^{-1}\left(\frac{1-s_{j}^{*}}{A^{*}}\left(y_{j h}+y_{j l}+P\right)\right)}{\frac{1-s_{j}^{*}}{A^{*}}\left(y_{j h}+y_{j l}\right)},
$$

and, therefore, $\varphi_{j}\left(\frac{1-s_{j}^{*}}{A^{*}}\left(y_{j h}+P\right)\right)<\frac{y_{j h}}{y_{j h}+y_{j l}} \varphi_{j}\left(\frac{1-s_{j}^{*}}{A^{*}}\left(y_{j h}+y_{j l}+P\right)\right)$.

If member $l$ also makes a positive contribution in equilibrium, we can similarly get $\varphi_{j}\left(\frac{1-s_{j}^{*}}{A^{*}}\left(y_{j l}+P\right)\right)<\frac{y_{j l}}{y_{j h}+y_{j l}} \varphi_{j}\left(\frac{1-s_{j}^{*}}{A^{*}}\left(y_{j h}+y_{j l}+P\right)\right)$. Hence, regardless of whether member $l$ is a non-contributor or not, we have

$$
\varphi_{j}\left(\frac{1-s_{j}^{*}}{A^{*}}\left(y_{j h}+P\right)\right)+\varphi_{j}\left(\frac{1-s_{j}^{*}}{A^{*}}\left(y_{j l}+P\right)\right)<\varphi_{j}\left(\frac{1-s_{j}^{*}}{A^{*}}\left(y_{j h}+y_{j l}+P\right)\right) .
$$

Let $v_{j h}^{v}=y_{j h}+y_{j l}+P, v_{j l}^{v}=P$, and $v_{j k}^{v}=v_{j k}$ for all $k \neq h, l$ be the new distribution of interests. By using the function $A_{j}\left(A, \mathbf{v}_{j}\right)$ defined in the proof of Proposition 1, we can then derive the inequality

$$
A_{j}\left(A^{*}, \mathbf{v}_{j}\right)=\sum_{k=1}^{N_{j}} \rho_{j k}\left(A^{*}, A_{j}\left(A^{*}, \mathbf{v}_{j}\right), v_{j k}\right)<\sum_{k=1}^{N_{i}} \rho_{j k}\left(A^{*}, A_{j}\left(A^{*}, \mathbf{v}_{j}\right), v_{j k}^{v}\right),
$$


which implies that $A_{j}\left(A^{*}, \mathbf{v}_{j}^{v}\right)>A_{j}\left(A^{*}, \mathbf{v}_{j}\right)$. The property (a) is now derived by using Lemma 1. As long as we find more than one member in group $i$ who has a positive share in the private-part of the prize, we can reapply this property. Hence property (b) follows.

Q.E.D.

\section{Proof of Proposition 3.}

In equilibrium, the expected payoff of member $k$ of group $j$ can be represented as the value of the following function at $v=v_{j k}$, pretending that $A^{*}$ and $s_{j}{ }^{*}$ are constants:

$$
E U^{*}(v)=s_{j}^{*} v-c_{j}\left(\varphi_{j}\left(\frac{1-s_{j}^{*}}{A^{*}} v\right)\right) \text {. }
$$

We can calculate the partial derivative

$$
\frac{\partial E U^{*}}{\partial v}=\frac{s_{j}^{*}}{\alpha_{j}\left(\varphi_{j}\left(\frac{1-s_{j}^{*}}{A^{*}} v\right)\right)} \cdot\left\{\alpha_{j}\left(\varphi_{j}\left(\frac{1-s_{j}^{*}}{A^{*}} v\right)\right)-\frac{1-s_{j}^{*}}{A_{j}^{*}} \varphi_{j}\left(\frac{1-s_{j}^{*}}{A^{*}} v\right)\right\},
$$

as long as $\varphi_{j}\left(\frac{1-s_{j}^{*}}{A^{*}} v\right)>0$.

Q.E.D.

\section{Proof of Corollary.}

Proposition 1 implies that there exists $A^{0}>0$ satisfying $s_{j}\left(A^{0}\right)=\frac{1}{2}$. Apply the regularity condition $R C$ to find $a^{0}>0$ such that $\frac{c_{j}^{\prime \prime}(a) a}{c_{j}^{\prime}(0)} \leq \frac{1}{2 N_{j}}$ for all $0<a \leq a^{0}$. Since $c_{j}^{\prime}(0)>0$, the function $A_{j}\left(A, \mathbf{v}_{j}\right)$ defined in the proof of Proposition 1 converges to 0 as $A$ goes to infinity. We can find $\bar{A}>0$ with $A_{j}\left(A, \mathbf{v}_{i}\right) \leq a^{0}$ for all $A \geq \bar{A}$. Let $A^{E}=\max \left\{\bar{A}, A^{0}\right\}$. If $A^{*}$ is larger than or equal to $A^{E}$, we get that

$$
\sup _{k} \alpha_{j}\left(\varphi_{j}\left(\frac{1-s_{j}^{*}}{A^{*}} v_{j k}\right)\right)<\frac{c^{\prime}\left(A_{j}\left(A^{*}, \mathbf{v}_{j}\right)\right) A_{j}\left(A^{*}, \mathbf{v}_{j}\right)}{c_{j}^{\prime}(0)} \leq \frac{1}{N_{j}}\left(1-s_{j}^{*}\right),
$$

which establishes inequality (6) for $\xi=0$.

Q.E.D.

\section{Proof of Proposition 4.}


If the marginal cost function $c_{j}^{\prime}$ is strictly convex (concave) and $c_{j}^{\prime}(0)=0$ holds, $\varphi_{j}$ is strictly concave (convex). We can follow Dasgupta et al. (1973) to show that a Lorenzworsening change in the stake vector $\mathbf{v}_{j}$ will cause a decrease (increase) in the aggregate contribution $\sum_{k=1}^{N_{j}} \varphi_{j}\left(\frac{A^{*}-A_{j}^{*}}{\left(A^{*}\right)^{2}} v_{j k}\right)$ of group $j$ for the initial equilibrium values $A^{*}$ and $A_{j}^{*}$. Then the value of the share function declines (increases) at $A^{*}$. The desired results can be derived with Lemma 1.

Q.E.D. 


\section{References}

Baik, Kyung H. 2008. “Contests with Group-Specific Public-Good Prizes.” Social Choice and Welfare, 30(1): 103-117.

Baik, Kyung H., and Sanghack Lee. 2007. "Collective Rent Seeking When Sharing Rules are Private Information.” European Journal of Political Economy, 23 (September): 768-76.

Bergstrom, Theodore, Lawrence Blume, and Hal Varian. 1986. "On the Private Provision of Public Goods.” Journal of Public Economics, 29(1): 25-49.

Cornes, Richard, and Roger Hartley. 2003. "Risk Aversion, Heterogeneity and Contests," Public Choice, 117(1): 1-25.

Cornes, Richard, and Roger Hartley. 2005. "Asymmetric Contests with General

Technologies." Economic Theory, 26(4): 923-946.

Dasgupta, Partha, Amartya Sen, and David Starrett. 1973. "Notes on the Measurement of Inequality." Journal of Economic Theory, 6(2): 180-187.

Epstein, Gil S., and Yosef Mealem. 2009. "Group Specific Public Goods, Orchestration of Interest Groups and Free Riding." Public Choice, 139(3-4): 357-369.

Esteban, Joan-Maria, and Debraj Ray. 1999. "Conflict and Distribution.” Journal of Economic Theory, 87(2): 379-415.

Esteban, Joan-Maria, and Debraj Ray. 2001. "Collective Action and the Group-Size Paradox." American Political Science Review, 95(3): 663-672.

Esteban, Joan-Maria, and Debraj Ray. 2011a. "Linking Conflict to Inequality and Polarization." American Economic Review, 101(4): 1345-1374.

Esteban, Joan-Maria, and Debraj Ray. 2011b. "A Model of Ethnic Conflict.” Journal of the European Economic Association. 9(3): 1496-1521.

Katz, Eliakim, Shmuel Nitzan, and Jacob Rosenberg. 1990. "Rent-Seeking for Pure Public Goods.” Public Choice, 65(April): 49-60.

Katz, Eliakim and Julia Tokatlidu. 1996. “Group Competition for Rents.” European Journal of Political Economy, 12(4): 599-607.

Konrad, Kai A. 2009. Strategy and Dynamics in Contests. New York: Oxford University Press.

Lee, Sanghack. 1995. "Endogenous sharing rules in collective-group rent-seeking." Public Choice, 85(1-2): 31-44. 
Niou, Emerson M. S., and Guofu Tan. 2005. "External Threat and Collective Action." Economic Inquiry, 43(3): 519-530.

Nitzan, Shmuel. 1991. "Collective Rent Dissipation.” Economic Journal, 101(409): 15221534.

Nitzan, Shmuel, and Kaoru Ueda. 2011. "Prize Sharing in Collective Contests." European Economic Review, 55(5), 678-687.

Olson, Mancur. 1965. The Logic of Collective Action. Cambridge, M.A.: Harvard University Press.

Olson, Mancur, and Richard Zeckhauser. 1966. "An Economic Theory of Alliances." Review of Economics and Statistics, 48(3): 266-279.

Riaz, Khalid, Jason F. Shogren and Stanley R. Johnson. 1995. "A General Model of Rent-Seeking for Public Goods." Public Choice, 82(March): 243-59.

Ryvkin, Dmitry. 2011 "The optimal sorting of players in contests between groups." Games and Economic Behavior, 73(2): 564-572.

Sandler, Todd. 1992. Collective Action. Ann Arbor: The University of Michigan Press. Sandler, Todd. 1993. "The Economic Theory of Alliances: A Survey." The Journal of Conflict Resolution, 37(3): 446-483.

Ueda, Kaoru. 2002. "Oligopolization in Collective Rent-Seeking." Social Choice and Welfare, 19(3): 613-626. 